\title{
Optimization of coronagraph design for segmented aperture telescopes
}

Jeffrey Jewell, Garreth Ruane, Stuart Shaklan, Dimitri Mawet, Dave Redding

Jeffrey Jewell, Garreth Ruane, Stuart Shaklan, Dimitri Mawet, Dave Redding, "Optimization of coronagraph design for segmented aperture telescopes," Proc. SPIE 10400, Techniques and Instrumentation for Detection of Exoplanets VIII, 104000H (13 September 2017); doi: 10.1117/12.2274574

SPIE Event: SPIE Optical Engineering + Applications, 2017, San Diego, California, United States 


\title{
Coronagraph Design Optimization for Segmented Aperture Telescopes
}

\author{
Jeffrey Jewell ${ }^{\mathrm{a}}$, Garreth Ruane ${ }^{\mathrm{b}}$, Stuart Shaklan ${ }^{\mathrm{a}}$, Dimitri Mawet ${ }^{\mathrm{b}}$, and Dave Redding ${ }^{\mathrm{a}}$ \\ a Jet Propulsion Laboratory, California Institute of Technology, 4800 Oak Grove Dr., Pasadena, \\ CA 91109, USA; \\ ${ }^{\mathrm{b}}$ Department of Astronomy, California Institute of Technology, 1200 E. California Blvd., \\ Pasadena, CA 91125, USA
}

\begin{abstract}
The goal of directly imaging Earth-like planets in the habitable zone of other stars has motivated the design of coronagraphs for use with large segmented aperture space telescopes. In order to achieve an optimal trade-off between planet light throughput and diffracted starlight suppression, we consider coronagraphs comprised of a stage of phase control implemented with deformable mirrors (or other optical elements), pupil plane apodization masks (gray scale or complex valued), and focal plane masks (either amplitude only or complex-valued, including phase only such as the vector vortex coronagraph). The optimization of these optical elements, with the goal of achieving 10 or more orders of magnitude in the suppression of on-axis (starlight) diffracted light, represents a challenging non-convex optimization problem with a nonlinear dependence on control degrees of freedom. We develop a new algorithmic approach to the design optimization problem, which we call the "Auxiliary Field Optimization" (AFO) algorithm. The central idea of the algorithm is to embed the original optimization problem, for either phase or amplitude (apodization) in various planes of the coronagraph, into a problem containing additional degrees of freedom, specifically fictitious "auxiliary" electric fields which serve as targets to inform the variation of our phase or amplitude parameters leading to good feasible designs. We present the algorithm, discuss details of its numerical implementation, and prove convergence to local minima of the objective function (here taken to be the intensity of the on-axis source in a "dark hole" region in the science focal plane). Finally, we present results showing application of the algorithm to both unobscured off-axis and obscured on-axis segmented telescope aperture designs. The application of the AFO algorithm to the coronagraph design problem has produced solutions which are capable of directly imaging planets in the habitable zone, provided end-to-end telescope system stability requirements can be met. Ongoing work includes advances of the AFO algorithm reported here to design in additional robustness to a resolved star, and other phase or amplitude aberrations to be encountered in a real segmented aperture space telescope.
\end{abstract}

Keywords: vortex coronagraph, optimization, deformable mirrors, apodization

\section{INTRODUCTION}

The goal of directly imaging Earth-like planets in the habitable zone of other stars in our galaxy (0.7 to 1.5 AU from the host star) has motivated the design of coronagraphs for use with large segmented aperture space telescopes. A star 10, 30, or 50 parsec away hosting a planet at $1 \mathrm{AU}$, will require a telescope approximately 3.4 , 10.2 , or 17.0 meters in diameter respectively to image (at a wavelength of $550 \mathrm{~nm}$ ) the planet in a focal plane at 3 diffraction limited "resolution elements" (or $3 \lambda / D$ ) from the on-axis star. Moreover, the star is 10 billion times brighter than the planet in reflected light. ${ }^{1}$ The goal of direct imaging of exoplanets in the habitable zone therefore requires large aperture space telescopes, in order to collect enough photons from the planet, as well as some method of high-contrast imaging, in order to suppress the diffracted starlight even as close as an Inner Working Angles (IWA) of $3 \lambda / D$. Achieving a direct imaging capability for exoplanets in the habitable zone therefore requires the development of high-contrast imaging coronagraphs or starshades designed to work with large, segmented aperture, space telescopes.

Further author information: send correspondence to Jeffrey.B.Jewell@jpl.nasa.gov

Techniques and Instrumentation for Detection of Exoplanets VIII, edited by Stuart Shaklan, Proc. of SPIE Vol. 10400, 104000H · C 2017 SPIE · CCC code: 0277-786X/17/\$18 · doi: 10.1117/12.2274574 
Optimal coronagraph designs must strike a balance between planet light throughput and diffracted starlight suppression. We have excellent coronagraph designs for simple, uniform circular apertures, such as a monolithic primary with an off-axis secondary mirror. However, we currently do not have the technological means to increase telescope primary mirror diameters to the size driven by exoplanet imaging science goals with uniform circular apertures, necessitating new coronagraph designs capable of $10^{-10}$ suppression of diffracted starlight from the additional structure in segmented apertures.

The coronagraphs we will consider are composed of two stages of phase control (in two planes, implemented with deformable mirrors or other devices), a focal plane mask, and a Lyot stop. General end to end optimization of a coronagraph represents a non-convex problem with a nonlinear dependence on control parameters. In this paper, we introduce a new algorithmic approach to the design optimization problem, which we call the "Auxiliary Field Optimization" (AFO) algorithm. The central idea of the algorithm is to embed the original optimization problem, for either phase or amplitude (apodization) in various planes of the coronagraph, into a problem containing additional degrees of freedom, specifically fictitious "auxiliary" electric fields which serve as targets to inform the variation of our phase or amplitude parameters leading to good feasible designs.

The results in this paper are with respect to optimization of the two phase controls (i.e. deformable mirrors), with the focal plane mask chosen to be a vortex phase only mask, ${ }^{2}$ and a Lyot plane binary mask, typically either an annulus or slightly undersized aperture mask. We have applied the AFO algorithm to solve for apodization gray-scale masks tailored for use with a vortex focal plane mask - for a detailed discussion of these apodozation solutions and sensitivity analysis we refer the reader to G.Ruane et al, "Performance and Sensitivity of Vortex Coronagraphs on Segmented Space Telescopes", in these proceedings, as well as a summary of apodization solutions for a catalog of potential segmented aperture designs. ${ }^{3}$ In this paper, we present the AFO algorithm, discuss details of its numerical implementation, and present results showing application to an unobscured fourring hex aperture (included in the Segmented Coroangaph Design and Analysis (SCDA) Program ${ }^{4,5}$ aperture catalog), as well as the LUVOIR architecture 'A' initial aperture design.

\section{THE CORONAGRAPH DESIGN OPTIMIZATION PROBLEM}

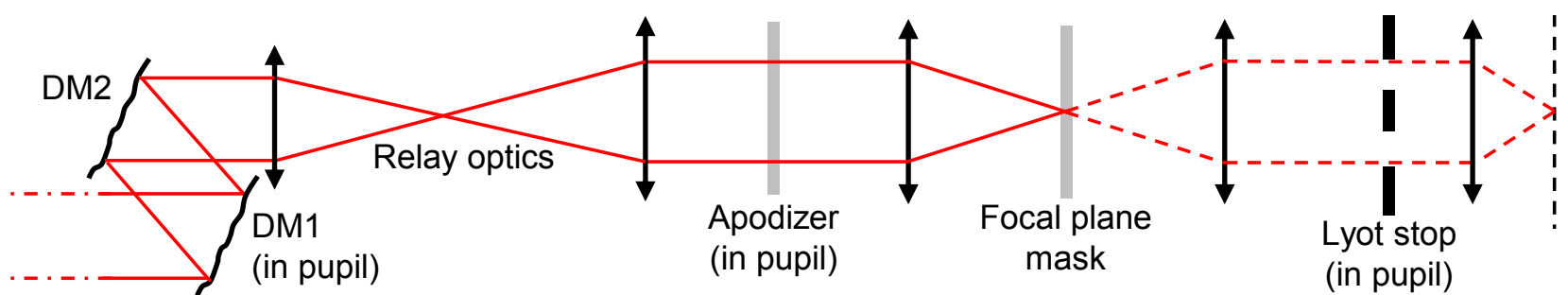

Figure 1. Coronagraph model and control degrees of freedom to be optimized for suppression of diffracted starlight.

In Fig. 1 we show a schematic of the coronagraph model used here. The model begins at an entrance pupil plane, where we have a deformable mirror for manipulation of the phase of the field. We then Fresnel propagate to another plane containing a second deformable mirror. We Fresnel propagate back to a conjugate pupil plane, after which we Fourier transform to a focal plane, where we have a mask (for this paper we use a vortex phase only mask). We then inverse Fourier transform to the Lyot plane where we use a binary Lyot stop, after which we Fourier transform the field to reach the final science focal plane. To fix notation, we represent the forward propagation of the entrance pupil electric field to the final science focal plane according to

$$
\mathcal{E}(\xi)=\left(F \Theta F^{\dagger} \Omega F\right)\left(P_{\lambda}^{\dagger} e^{i \Gamma\left(\lambda_{0} / \lambda\right)} P_{\lambda} e^{i \Phi\left(\lambda_{0} / \lambda\right)}\right) X(\xi)
$$

where the various optical elements are: 
1. Entrance pupil electric field $X(\xi)$ (parametrized by the location $\xi$ of the source in the focal plane). In this notation the on-axis source (i.e. the star) is $X(0)$, and assumed to be a re-imaged version of the telescope primary aperture in the coronagraph entrance pupil.

2. Entrance pupil phase control degrees of freedom (implemented by an optical element such as a deformable mirror) represented as $e^{i \Phi\left(\lambda_{0} / \lambda\right)}$. In this notation, we mean that the phase $\Phi$ has some value at every pixel in the entrance pupil, and $e^{i \Phi\left(\lambda_{0} / \lambda\right)}$ represents the complex-valued phase factor multiplying the incident electric field $X(\xi)$.

3. Fresnel propagation, denoted $P_{\lambda}$, from the entrance pupil to what we call the "Fresnel plane" for purposes of discussion in what follows.

4. Fresnel plane phase control degrees of freedom represented as $e^{i \Gamma\left(\lambda_{0} / \lambda\right)}$.

5. Fresnel propagation back to a reference conjugate pupil plane, denoted $P_{\lambda}^{\dagger}$.

6. Fourier transform, denoted $F$ to a focal plane.

7. Multiplication of the incident field in the focal plane with a focal plane mask denoted $\Omega$. For the purposes of this paper, we will fix $\Omega$ to be a vortex phase only focal plane mask $e^{i l \phi}$, with $\phi$ the polar angle in cylindrical coordinates in the focal plane, and $l$ the integer "topological chage".

8. Inverse Fourer transform to the Lyot (pupil) plane, with Lyot stop mask $\Theta$.

9. A final Fourier transfrom to the science focal plane.

For convenience in what follows, we also denote the linear operator mapping the entrance pupil to the conjugate pupil (given the entrance pupil phase $\Phi$ and the Fresnel plane phase $\Gamma$ )

$$
U_{\lambda}(\Gamma, \Phi)=P_{\lambda}^{\dagger} e^{i \Gamma\left(\lambda_{0} / \lambda\right)} P_{\lambda} e^{i \Phi\left(\lambda_{0} / \lambda\right)}
$$

as well as the linear operator mapping the conjugate pupil plane to the science focal plane

$$
C=F \Theta F^{\dagger} \Omega F
$$

In this notation, the science focal plane field for the on-axis source is compactly written as $\mathcal{E}=C U(\Gamma, \Phi) X(0)$.

In the case of a uniform circular aperture, a vortex coronagraph with slightly undersized outer radius Lyot stop provides perfect suppression of an on-axis point source. ${ }^{2}$ As discussed earlier however, the drive to larger telescopes for exoplanet imaging requires segmented telescopes, resulting in diffraction from the additional structure of segment gaps and also the secondary mirror and support struts. This diffracted light makes the vortex unusable for segmented aperture telescopes - our problem then is to optimize either phase corrections in the entrance and Fresnel planes, and / or apodization in the conjugate pupil plane in order to suppress the diffracted light due to structure in a segmented aperture.

Specifically, the design optimization problem is to minimize the energy from the star in a "dark hole region" in the science focal plane where we expect a planet might be located. Denoting the science focal plane dark hole region by $Q$ (an annular binary mask specified by an Inner and Outer Working Angle), our goal is to find coronagraph design variables,

$$
(\hat{\Gamma}, \hat{\Phi})=\min _{\Gamma, \Phi}\|Q C U(\Gamma, \Phi) X(0)\|^{2}
$$

We now discuss a novel algorithm developed to solve this optimization problem, and show the resulting solutions enabling a vortex to work with large segmented aperture telescopes. 


\section{AUXILIARY FIELD ALGORITHM FOR CORONAGRAPH OPTIMIZATION}

Coronagraph optimization is a non-convex optimization problem with a nonlinear dependence on parameters. The non-convex nature of the problem is due to the coronagraph architecture - we have control degrees of freedom in different planes, leading to the structure of a product of (a parametric family of) linear operators. The nonlinear nature of the problem is due to the fact that we use phase control, and so have a complex exponential dependence of the objective on our control degrees of freedom.

One approach to the minimization of $\|Q C U(\Gamma, \Phi) X(0)\|^{2}$ is gradient descent and related approaches (conjugate gradient descent, or variable metric). These methods compute the direction resulting in a steep local decrease of the function, followed by a line search along this direction resulting in a minimum. However, the nonlinear dependence on phase degrees of freedom results in a computationally expensive line search. Other sub-optimal criteria might be used (for example a backtracking line search terminating based on the ArmijoGoldstein condition ${ }^{6}$ ). In addition to computationally expensive line searches, gradient descent techniques can result in local minima solutions, requiring other tricks to jump out and eventually settle on good coronagraph designs.

Another strategy for coronagraph optimization is to look for a way to introduce convex, linear sub-problems as a way of informing the optimization of the nonlinear degrees of freedom. Recent applications of stroke minimization Electric Field Conjugation $(\mathrm{EFC})^{7}$ are an example of this family of algorithm. This method introduces a linear approximation of the nonlinear phase control directly in the objective function, and then solves for the optimal phase variation. Assuming the deformable mirror phase is modeled as a linear combination of basis functions $H a$ (with the columns of the matrix $H$ given by the DM influence functions, and $a$ the column vector of basis function coefficients representing actuator heights), the approximation, for small phase variations

$$
e^{i H(a+\delta)} \approx e^{i H a}(1+i H \delta)
$$

leads to a linearized form for the perturbation to the focal plane electric field

$$
\mathcal{E} \approx \mathcal{E}_{0}+S \delta
$$

where $S$ is the "sensitivity matrix" (the local Jacobian of the focal plane field with respect to the phase control degrees of freedom in either the entrance pupil plane or Fresnel plane). Substituting this approximation into the objective function leads to the linear subproblem

$$
\begin{aligned}
\hat{\delta} & =\min _{\delta}\left(\left\|\mathcal{E}_{0}+S \delta\right\|^{2}+\mu\|\delta\|^{2}\right) \\
& =\left(\mu I+S^{\dagger} S\right)^{-1}(-1) S^{\dagger} \mathcal{E}_{0}
\end{aligned}
$$

The complication with the above is that for coronagraph optimization with segmented apertures, the dynamic range of contrast we need to achieve requires DM variations that are well outside the validity of the linear approximation above. We are therefore forced to recompute the sensitivity matrix $S$ about the current DM solutions frequently as we optimize. ${ }^{8}$

Given the computational expense for both gradient descent line searches and recomputing the Jacobian for the focal plane field with respect to phase degrees of freedom, we look for alternative algorithms utilizing convex linear subproblems informing the variations of nonlinear control degrees of freedom. We begin with the question: "What (conjugate pupil) field would result in vanishing energy in the dark hole region?" It is interesting to consider such a set of conjugate pupil fields since the field in the Fresnel or entrance pupil can then be immediately computed within our model by propagating "backwards" (possible since the Fresnel propagators are invertible; in fact unitary operators with an inverse given by their adjoint). This would suggest that adjusting the phase control degrees of freedom can be done almost analytically given a specific target field propagated back from the conjugate pupil.

Of course, there are many possible conjugate pupil fields which give vanishing energy from the on-axis source in the dark hole region. We immediately recognize the need to regularize our choice. One obvious regularization is to penalize the conjugate pupil field from being too far away from the actual physical field incident on the 
conjugate pupil. If we begin with flat DM's (no phase wavefront control), we have a one dimensional parametric family of conjugate pupil plane fields, depending on a parameter " $b$ ", striking a balance between vanishing dark hole energy for the on-axis source and not deviating too far from the actual physical conjugate field,

$$
\hat{W}=\min _{W}\left(\|Q C W\|^{2}+b\|W-X(0)\|^{2}\right)
$$

(with the entrance pupil aperture mask $X(0)$ set by the telescope design). The solution to this problem is

$$
\hat{W}=\left(b I+C^{\dagger} Q C\right)^{-1} b X(0)
$$

(where $C^{\dagger}$ is the conjugate transpose of the coronagraph operator $C$ ), and represent a one-parameter family of target fields depending on the regularization parameter ' $b$ '. We will discuss details of solving this (linear) problem in what follows, as well as prove that the target field, in the limit $\lim _{b \rightarrow 0}$, has vanishing energy in the dark hole region We show such an example target field in Fig. 2, computed for the LUVOIR mission concept architecture 'A' telescope design, with a charge 6 vortex focal plane mask and a simple annular Lyot stop downstream of the conjugate pupil.

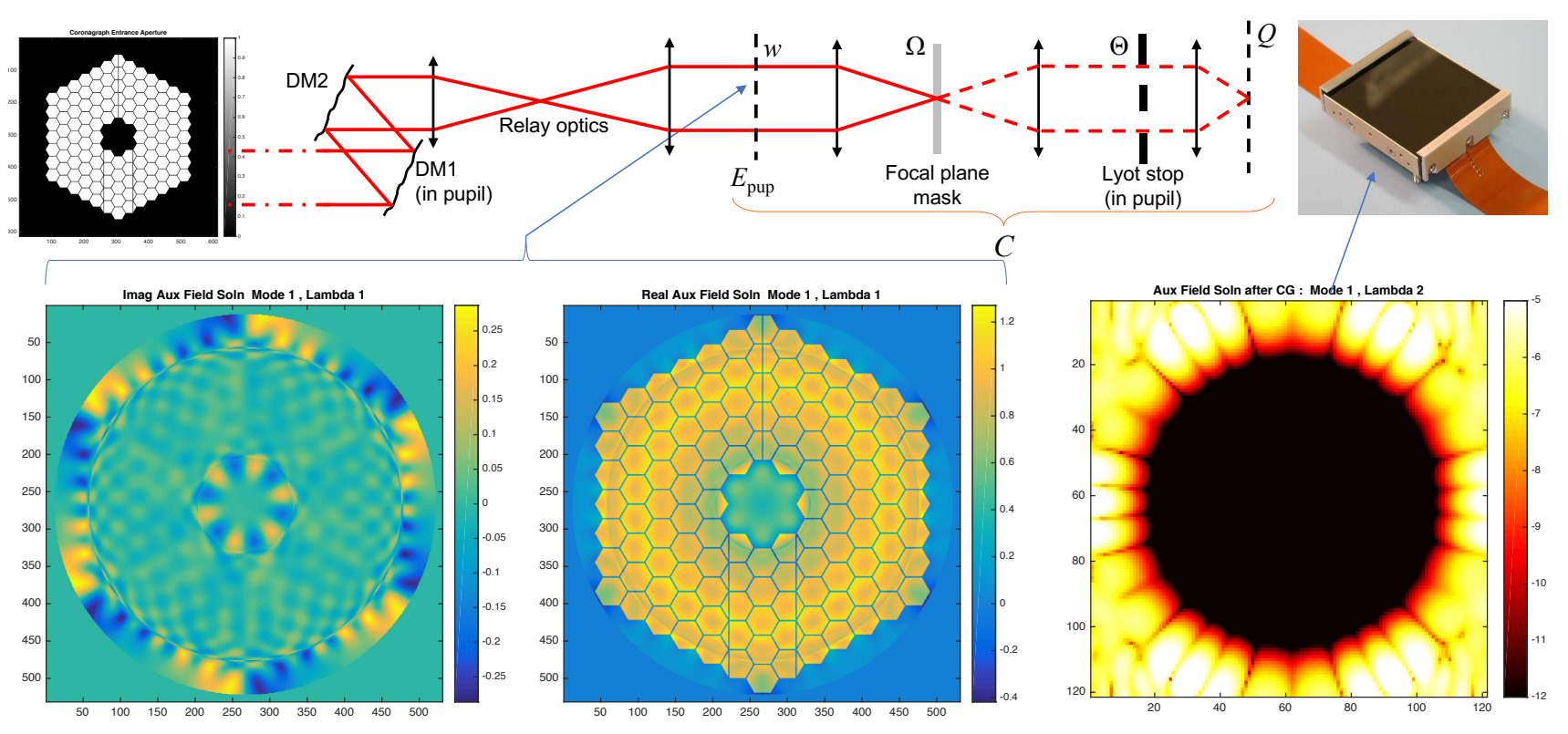

Figure 2. The imaginary (lower left) and real (lower middle) parts of the target (conjugate plane) complex electric field for the on-axis source has $\lim _{b \rightarrow 0}\|Q C W\|^{2}=0$ energy in the dark hole region where planets live!

As this fictitious conjugate pupil field $W$ has vanishing dark hole energy, it is an ideal target to try to "hit" by adjusting our phase control degrees of freedom. We do so by next solving for the phase degrees of freedom which best approximate $W$,

$$
\{\Gamma, \Phi\}=\min _{\Gamma, \Phi}\left(\left\|W-\left(P_{\lambda}^{\dagger} e^{i\left(\lambda_{0} / \lambda\right) \Gamma} P_{\lambda} e^{i\left(\lambda_{0} / \lambda\right) \Phi}\right) X(0)\right\|^{2}\right)
$$

While this is a nonlinear, non-convex optimization subproblem, the unitary (and invertible) nature of the Fresnel propagators, allow accurate, and efficient, solutions to this subproblem by alternating minimization of one DM with the other fixed, and back-propagating the target field to the plane being optimized (details to be discussed in what follows).

Our new algorithmic approach is to simply iterate these two steps, where given our current DM phase control solution we solve for the target field $W$, and with that ideal conjugate pupil field, we adjust the DM's to best 
approximate that target. In what follows we will refer to this algorithm as the "Auxiliary Field Optimization" (AFO) algorithm (the name referring to the embedding of the original optimization problem for the phase control degrees of freedom into a larger dimensional problem including the fictitious target conjugate pupil plane field $W$ as "auxiliary variables" to help inform the choice of phase variations).

It is possible to show that this algorithm, based on the above simple intuition, can be proven to converge to a local minimum of our objective (as discussed in detail in Appendix A.2). "Under the hood", the algorithm is a variant of an "Expectation-Maximization" algorithm, ${ }^{9}$ and hints at the value of a probabilistic setting for constructing coronagraph design optimization algorithms. It is beyond the scope of this paper to explore probabilistic or random search ideas further, but we note simply that such algorithms (such as simulated annealing) are among the few capable of delivering global optimal solutions to non-convex problems, although at potentially tremendous computational expense. We simply use a probabilistic framework here to formulate a simpler, deterministic algorithm (capable of only "local minimum" guarantees). We now proceed to discuss the numerical implementation of the algorithm and then show results on various segmented aperture telescope designs.

\section{NUMERICAL IMPLEMENTATION OF THE AUXILIARY FIELD ALGORITHM}

\subsection{Solving for the Target Field}

As noted above, the first step of an iterative cycle of the AFO is to solve for an updated target conjugate pupil field

$$
\begin{aligned}
\hat{W} & =\min _{W}\left(\|Q C W\|^{2}+b\|\hat{W}-U(\Gamma, \Phi) X(0)\|^{2}\right) \\
& =\left(b I+C^{\dagger} Q C\right)^{-1} b U(\Gamma, \Phi) X(0)
\end{aligned}
$$

While this is a linear problem, it is non-trivial to solve. For numerical accuracy, it is common to use $\sim 1000$, or $\sim 2000$ pixels across the entrance pupil aperture, and further zero pad to give sufficient sampling in subsequent focal planes in the coronagraph. As we have identified the conjugate pupil as a convenient place to solve for an ideal field, the matrix to be inverted $\left(b I+C^{\dagger} Q C\right)^{-1}$ is $\sim 10^{6} \times 10^{6}$ even if restricted to a region in the conjugate pupil matched to the support of the entrance pupil. A matrix of this size is prohibitive to store let alone compute by brute force and invert.

However, we recall the "Sherman-Woodbury" matrix identity for the inverse of this matrix,

$$
\left(b I+C^{\dagger} Q C\right)^{-1}=\left[I-C^{\dagger} Q\left(b I+Q C C^{\dagger} Q\right)^{-1} Q C\right]
$$

The significance of this identity is two-fold: 1) we can multiply by the "forward" and "adjoint" coronagraph operators using forward and inverse Fourier transforms and multiplication by the vortex focal plane (or its complex conjugate) respectively, and 2) the "internal" matrix $\left(b I+Q C C^{\dagger} Q\right)^{-1}$ is of a size $N_{D H} \times N_{D H}$, with $N_{D H}$ the number of pixels in the science focal plane dark hole region. This is a much smaller matrix, typically $10^{4} \times 10^{4}$ for focal plane sampling of 4 pixels $/(\lambda / D)$, and inner and outer working angles of $3 \lambda / D$ and $10 \lambda / D$ respectively.

Our solution for the auxiliary field, using the matrix identity above is therefore

$$
\hat{W}=\left[I-C^{\dagger} Q\left(b I+Q C C^{\dagger} Q\right)^{-1} Q C\right]\left(P^{\dagger} e^{i \Gamma} P e^{i \Phi} X(0)\right)
$$

We proceed to solve this by

1. Compute the true conjugate pupil field for the on-axis source $X(0)$

$$
Y_{1}=P^{\dagger} e^{i \Gamma} P e^{i \Phi} X(0)
$$

(using Fresnel propagation and operating with the current DM phase solutions). 
2. Send the conjugate pupil field through the coronagraph (through the vortex and Lyot stop to the science focal plane), by computing

$$
Y_{2}=\left(F \Theta F^{\dagger} \Omega F\right) Y_{1}
$$

(with $F$ and $F^{\dagger}$ forward and inverse Fourier transforms respectively, $\Omega$ the phase only vortex focal plane mask, and $\Theta$ the Lyot stop).

3. Keep only the forward field in the dark hole region by multiplying by the binary dark hole region mask $Y_{3}=Q Y_{2}$.

4. Solve the linear problem restricted to the dark hole region,

$$
Y_{4}=\left(b I+Q C C^{\dagger} Q\right)^{-1} Y_{3}
$$

5. Operate with the adjoint coronagraph operator (a linear mapping from the science focal plane to the conjugate pupil plane),

$$
Y_{5}=\left(F^{\dagger} \Omega^{*} F \Theta^{*} F^{\dagger}\right) Y_{4}
$$

(where $\Omega^{*}$ and $\Theta^{*}$ are the complex conjugates of the vortex focal plane and Lyot stop masks respectively).

6. Subtract from the true conjugate pupil field the correction $Y_{5}$ to solve for the new target auxiliary field

$$
\hat{W}=\left(P^{\dagger} e^{i \Gamma} P e^{i \Phi} A\right)-Y_{5}
$$

We refer to Equation 16 above as the "internal AFO linear problem", and we solve this by brute force having precomputed and stored a singular value decomposition of the matrix $Q C C^{\dagger} Q \equiv U \operatorname{Diag}(S) U^{\dagger}$, with the columns of $U$ the eigenmodes of the matrix $Q C C^{\dagger} Q$.

We now stress the physical importance of the eigenmodes of the internal AFO matrix used in explicitly solving Eequation 16 above, and show that the auxiliary field $W$ in the limit of very small regularization parameter $b \ll 1$ gives vanishing energy in the dark hole. To review, the eigenmodes satisfy the linear equation

$$
Q C C^{\dagger} Q\left|f_{j}\right\rangle=\left|\alpha_{j}\right|^{2}\left|f_{j}\right\rangle
$$

where the eigenvalues are deliberately written as the modulus squared of a complex number $\left|\alpha_{j}\right|^{2}=\alpha_{j}^{*} \alpha_{j}$ for reasons which will become clear. In the above we are borrowing a notation inherited from Quantum Mechanics to efficiently keep track of generally complex eigenmodes, where $\left|f_{j}\right\rangle$ can abstractly be thought of as a (complex valued) "column vector" and $\left\langle f_{j}\right|$ be thought of as a "row vector". The notation is also convenient as it denotes the eigenmodes as "vectors" in a Hilbert space of functions without explicit reference to basis. While working numerically, we will represent these modes at each "pixel" of the plane in which these modes live, i.e. focal plane or pupil plane. The eigenmodes form an orthogonal basis set, which means the inner products satisfy

$$
\left\langle f_{j} \mid f_{k}\right\rangle=\begin{array}{ll}
1 & \text { for } j=k \\
0 & \text { otherwise }
\end{array}
$$

We emphasize that the eigenmodes $\left|f_{j}\right\rangle$ have support in the dark region (they vanish completely outside the dark hole). We consider, for each focal plane dark hole eigenmode, as associated pupil plane mode

$$
C^{\dagger} Q\left|f_{j}\right\rangle=\alpha_{j}\left|g_{j}\right\rangle
$$

We investigate the properties of these pupil plane modes. First we compute the inner product of any two of these modes, and find they are orthogonal

$$
\begin{aligned}
\alpha_{j}^{*} \alpha_{k}\left\langle g_{j} \mid g_{k}\right\rangle & =\left\langle C^{\dagger} Q f_{j} \mid C^{\dagger} Q f_{k}\right\rangle \\
& =\left\langle f_{j}\left|\left(C^{\dagger} Q\right)^{\dagger} C^{\dagger} Q\right| f_{k}\right\rangle \\
& =\left\langle f_{j}\left|Q C C^{\dagger} Q\right| f_{k}\right\rangle \\
& =\left|\alpha_{k}\right|^{2}\left\langle f_{j} \mid f_{k}\right\rangle \\
& =\left|\alpha_{k}\right|^{2} \delta_{j k}
\end{aligned}
$$


This shows that $\left\langle g_{j} \mid g_{k}\right\rangle=\delta_{j k}$, and the associated pupil plane modes $\left|g_{j}\right\rangle$ form an orthogonal basis for pupil plane modes which directly contribute energy to the dark hole region. Finally we can show that the pupil plane modes also generate the original focal plane eigenmodes. Operating on both sides of the defining equation for the pupil plane modes 21 with $Q C$ we have

$$
\begin{aligned}
Q C\left(C^{\dagger} Q\left|f_{j}\right\rangle\right) & =\alpha_{j}(Q C)\left|g_{j}\right\rangle \\
& =\alpha_{j}^{*} \alpha_{j}\left|f_{j}\right\rangle
\end{aligned}
$$

or simply that

$$
Q C\left|g_{j}\right\rangle=\alpha_{j}^{*}\left|f_{j}\right\rangle
$$

This shows that the entire action of the vortex and Lyot stop in mapping a conjugate pupil field to the dark hole region can be compactly written in terms of the "input-output" response,

$$
Q C=\sum_{j} \alpha_{k}^{*}\left|f_{j}\right\rangle\left\langle g_{j}\right|
$$

while the adjoint linear operator mapping the dark hole region to an associated conjugate pupil plane is given by

$$
C^{\dagger} Q=\sum_{k} \alpha_{k}\left|g_{k}\right\rangle\left\langle f_{k}\right|
$$

and of course, with this "factorization" we recover our original eigenmode representation

$$
\begin{aligned}
Q C C^{\dagger} Q & =\left(\sum_{j} \alpha_{j}^{*}\left|f_{j}\right\rangle\left\langle g_{j}\right|\right)\left(\sum_{k} \alpha_{k}\left|g_{k}\right\rangle\left\langle f_{k}\right|\right) \\
& =\sum_{j k} \alpha_{j}^{*} \alpha_{k}\left|f_{j}\right\rangle\left\langle g_{j} \mid g_{k}\right\rangle\left\langle f_{k}\right| \\
& =\sum_{j}\left|\alpha_{j}\right|^{2}\left|f_{j}\right\rangle\left\langle f_{k}\right|
\end{aligned}
$$

(where in the last line we used the orthogonality relation $\left\langle g_{j} \mid g_{k}\right\rangle=\delta_{j k}$ ).

When viewed in terms of this eigenmode decomposition of the linear mapping from conjugate to science focal plane, one quickly sees that our implicit goal for the phase control is to somehow orthogonalize the on-axis source in the entrance pupil from the set of dark hole conjugate pupil eigenmodes. The significance of the auxiliary field is that, in the limit of vanishing regularization $b \ll 1$, it is exactly a projection of the on-axis source into the null space of $Q C$, giving zero energy in the dark hole, since we can show the solution is given in terms of the eigenmodes $\left|g_{j}\right\rangle$

$$
|\hat{W}\rangle=|U(\Gamma, \Phi) A\rangle-\left(\sum_{j}\left|g_{j}\right\rangle\left(\frac{\left|\alpha_{j}\right|^{2}}{b+\left|\alpha_{j}\right|^{2}}\right)\left\langle g_{j} \mid U(\Gamma, \Phi) X(0)\right\rangle\right)
$$

As $b \rightarrow 0$, the above perfectly vanishes for all the pupil modes $\left|g_{j}\right\rangle$ that map to the focal plane dark hole (once passed through the coronagraph $C$ ). We remind the reader of Figure 2, showing exactly this property.

\subsection{Variation of Phase}

We now discuss the second step of the AFO iteration, in which we adjust the DM phase solutions to best match the updated auxiliary field $W$ (itself a function of the previous DM solution estimate). With the updated auxiliary field in hand, we want to minimize the approximation error between the true conjugate pupil field and the target by solving Equation 10. This is still a hard problem as it is a non-convex subproblem with a nonlinear 
dependence on parameters. For this step, we alternate a minimization of one DM with the other fixed, which results at least in a reduction of approximation error.

We make the choice to first optimize the entrance pupil phase $\Phi$ given the target auxiliary field while holding the Fresnel plane DM phase $\Gamma$ fixed, i.e. we want to solve

$$
\delta \Phi=\min _{\delta \Phi}\left(\int d \lambda\left\|W_{\lambda}-P_{\lambda}^{\dagger} e^{i \Gamma\left(\lambda_{0} / \lambda\right)} P_{\lambda} e^{i \Phi\left(\lambda_{0} / \lambda\right)} e^{i \delta \Phi\left(\lambda_{0} / \lambda\right)} X(0)\right\|^{2}\right)
$$

It is here that we appreciate one of the motivations for solving for the auxiliary field in the conjugate pupil plane - we can take advantage of the unitary nature of the Fresnel propagators, giving their inverse simply as their conjugate transpose (or adjoint), $P^{-1}=P^{\dagger}$. The adjoint of the Fresnel propagator is easily shown to be the Fresnel propagator with a reversed sign of the distance of propagation along the optical axis. This allows us to back-propagate the target auxiliary field to either the Fresnel plane or entrance pupil plane, where we can almost analytically find the variation in phase required to hit the back-propagated target.

To make this explicit in solving the entrance pupil phase variation in Equation 29, we form the backpropagated entrance pupil target auxiliary field

$$
Z_{\lambda}(\Gamma)=e^{-i \Phi\left(\lambda_{0} / \lambda\right)} P_{\lambda}^{\dagger} e^{-i \Gamma\left(\lambda_{0} / \lambda\right)} P_{\lambda} W
$$

Because our approximation error metric is chosen with respect to the inner-product norm, unitary operators can be absorbed and we have exactly

$$
\begin{aligned}
\delta \Phi & =\min _{\delta \Phi}\left(\int d \lambda\left\|W_{\lambda}-P_{\lambda}^{\dagger} e^{i \Gamma\left(\lambda_{0} / \lambda\right)} P_{\lambda} e^{i \Phi\left(\lambda_{0} / \lambda\right)} e^{i \delta \Phi\left(\lambda_{0} / \lambda\right)} X(0)\right\|^{2}\right) \\
& =\min _{\delta \Phi}\left[\int d \lambda\left(Z_{\lambda}^{*}(\Gamma)-e^{-i \delta \Phi\left(\lambda_{0} / \lambda\right)} X^{*}(0)\right)\left(Z_{\lambda}(\Gamma)-e^{i \delta \Phi\left(\lambda_{0} / \lambda\right)} X(0)\right)\right]
\end{aligned}
$$

A variation in $\delta \Phi$ leads to the condition

$$
0=\int d \lambda\left(\frac{\lambda_{0}}{\lambda}\right)\left(Z_{\lambda}(\Gamma) e^{-i \delta \Phi\left(\lambda_{0} / \lambda\right)} X^{*}(0)-Z_{\lambda}^{*}(\Gamma) e^{i \delta \Phi\left(\lambda_{0} / \lambda\right)} X(0)\right)
$$

We define the modulus and phase at each wavelength

$$
Z_{\lambda}(\Gamma) X^{*}(0) \equiv\left|Z_{\lambda}(\Gamma) X^{*}(0)\right| e^{i \Psi_{\lambda}}
$$

so that the phase variation minimum satisfies

$$
\begin{aligned}
0 & =\int d \lambda\left(\frac{\lambda_{0}}{\lambda}\right)\left|Z_{\lambda}(\Gamma) X^{*}(0)\right|\left(e^{+i\left(\Psi_{\lambda}-\delta \Phi\left(\lambda_{0} / \lambda\right)\right)}-e^{-i\left(\Psi_{\lambda}-\delta \Phi\left(\lambda_{0} / \lambda\right)\right)}\right) \\
& =2 i \int d \lambda\left(\frac{\lambda_{0}}{\lambda}\right)\left|Z_{\lambda}(\Gamma) X^{*}(0)\right|\left(\frac{e^{+i\left(\Psi_{\lambda}-\delta \Phi\left(\lambda_{0} / \lambda\right)\right)}-e^{-i\left(\Psi_{\lambda}-\delta \Phi\left(\lambda_{0} / \lambda\right)\right)}}{2 i}\right) \\
& =2 i \int d \lambda\left(\frac{\lambda_{0}}{\lambda}\right)\left|Z_{\lambda}(\Gamma) X^{*}(0)\right| \sin \left[\Psi_{\lambda}-\delta \Phi\left(\lambda_{0} / \lambda\right)\right]
\end{aligned}
$$

Notice that for single wavelengths, we set the update in $\delta \Phi$ to $\Psi_{\lambda}$, which makes any monochromatic form above vanish. However, for any finite bandpass we only have the one DM or phase screen surface to minimize the above. Assuming the validity of a small angle approximation $\sin x \approx x$, we solve for an approximate phase variation

$$
\begin{aligned}
0 & =\int d \lambda\left(\frac{\lambda_{0}}{\lambda}\right)\left|Z_{\lambda}(\Gamma) X^{*}(0)\right| \sin \left[\Psi_{\lambda}-\delta \Phi\left(\lambda_{0} / \lambda\right)\right] \\
& \approx \int d \lambda\left(\frac{\lambda_{0}}{\lambda}\right) \mid Z_{\lambda}(\Gamma) X^{*}\left((0) \mid\left[\Psi_{\lambda}-\delta \Phi\left(\lambda_{0} / \lambda\right)\right]\right.
\end{aligned}
$$


giving the update to the phase

$$
\delta \Phi=\frac{\int d \lambda\left(\frac{\lambda_{0}}{\lambda}\right)\left|Z_{\lambda}(\Gamma) X^{*}(0)\right| \Psi_{\lambda}}{\int d \lambda\left(\frac{\lambda_{0}}{\lambda}\right)^{2}\left|Z_{\lambda}(\Gamma) X^{*}(0)\right|}
$$

The same procedure is then applied to solve for a variation in the DM in the Fresnel plane with the new entrance pupil phase $\Phi+\delta \Phi$ fixed.

We comment on the use of the small angle approximation above in Equation 35. This is not the same as a linearization of the DM action on an incident field. It assumes that the resulting approximation error (due to the fact that our DM has an assumed optical path difference wavelength dependence) to the ideal wavelength dependent phase variation $\Psi_{\lambda}$ is small. This is clearly the case for small enough band. In practice we have found this approximate method of updating the phase to work well for $10 \%$ bandwidths. We emphasize here that future refinements of the AFO algorithm can include more sophisticated solutions to the phase optimization subproblem (eqn. 29), possibly including gradient descent or other iterative approach, while taking advantage of the fast back-propagation of the target field in equation 30.

We note that while we could compute several cycles of phase variation above, we have found that in practice one cycle followed by another update in the target auxiliary field suffices to give convergence to phase solutions capable of $10^{-10}$ contrast levels over $10 \%$ bandwidths. Finally, we refer the reader to Appendix B for a generalization of the phase updates above when solving explicitly for coefficients in some set of DM "influence functions".

\section{APPLICATION TO THE SCDA AND LUVOIR APERTURES}

We have applied the AFO algorithm to a variety of potential segmented telescope aperture designs considered in the Segmented Coronagraph Design and Analysis (SCDA) study. ${ }^{4,5}$ Examples of possible segmented apertures considered at shown in Figure 3. The AFO algorithm resulted in both beam-shaping and apodization solutions using a vortex focal plane mask and simple Lyot stop, with $10^{-10}$ on-axis source diffracted light suppression, with excellent throughput. We now show in detail the contrast and throughout for both an off-axis, unobscured segmented aperture, as well as well as for the LUVOIR architecture 'A' design.

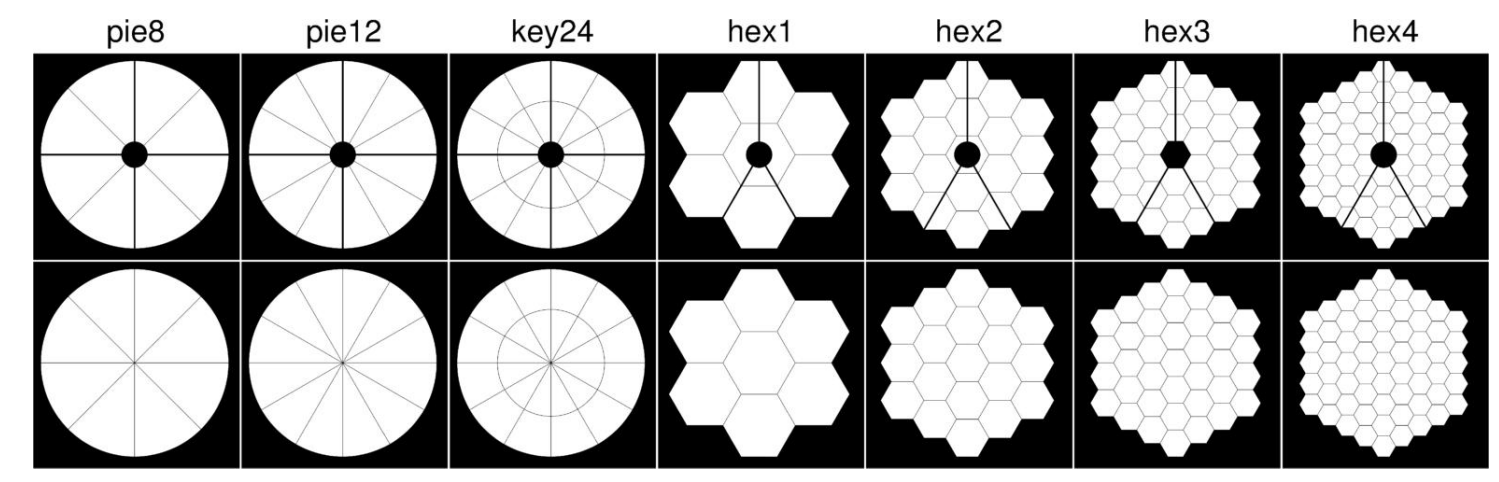

Figure 3. Example apertures used in the Segmented Coronagraph Design and Analysis (SCDA) study ${ }^{4,5}$ supported by the Exoplanet Exploration Program (ExEP).

\subsection{Unobstructed Apertures}

In Figures 4 and 5, we show the resulting solutions for beam shaping to an unobstructed four-ring Hex aperture. A charge 6 vortex focal plane mask was used with a simple uniform circular Lyot stop with an outer radius 0.99 with respect to the outer radius of the entrance pupil aperture. A DM array with actuator pitch of $400 \times 10^{-6}$ meters was used in the model, with localized DM influence functions. The DM solutions were optimized over a $10 \%$ band to suppress an on-axis point source. 

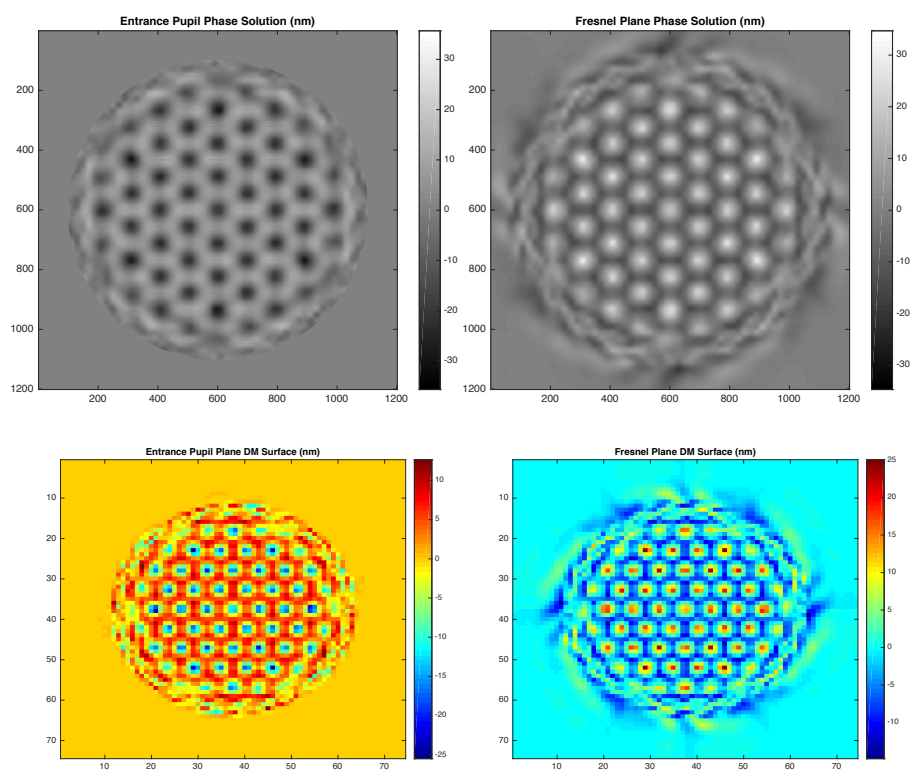

Figure 4. Deformable Mirror solutions for an unobstructed hex aperture. Upper left and right show the resulting phase applied on the Entrance Pupil and Fresnel Plane Deformable Mirror respectively, while the lower left and right show the actuator heights in nanometers.
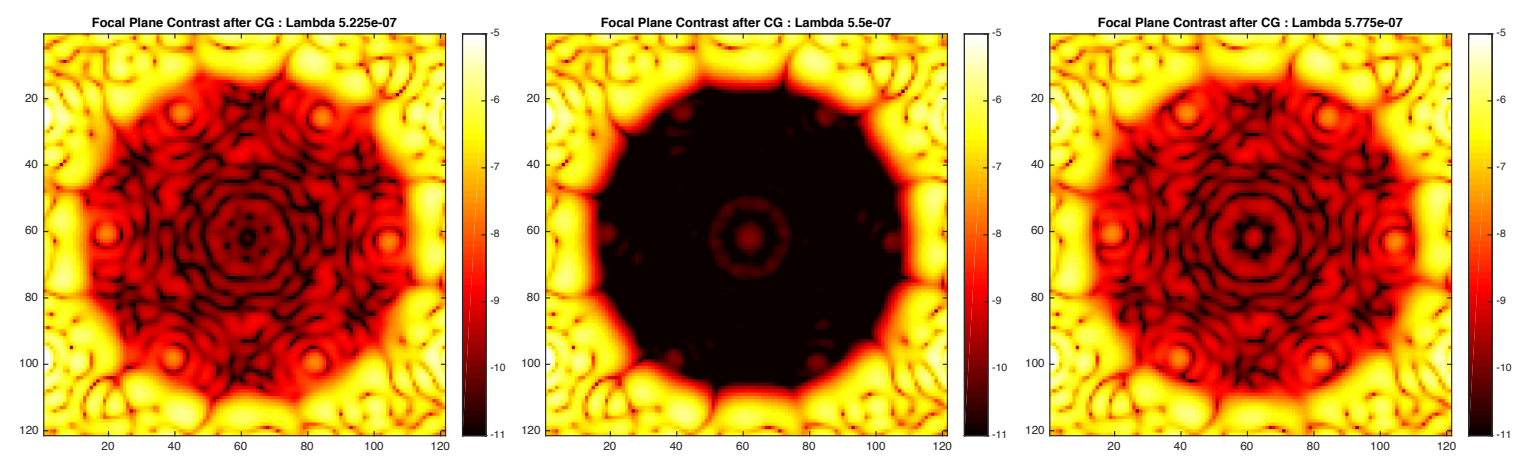

Figure 5. Science focal plane normalized intensity contrast for the DM solutions in Figure 4, at 522.5, 550, and 577.5 nanometers spanning a $10 \%$ bandpass. Bandpass averaged contrast achieved is $2.8 \times 10^{-10}$.

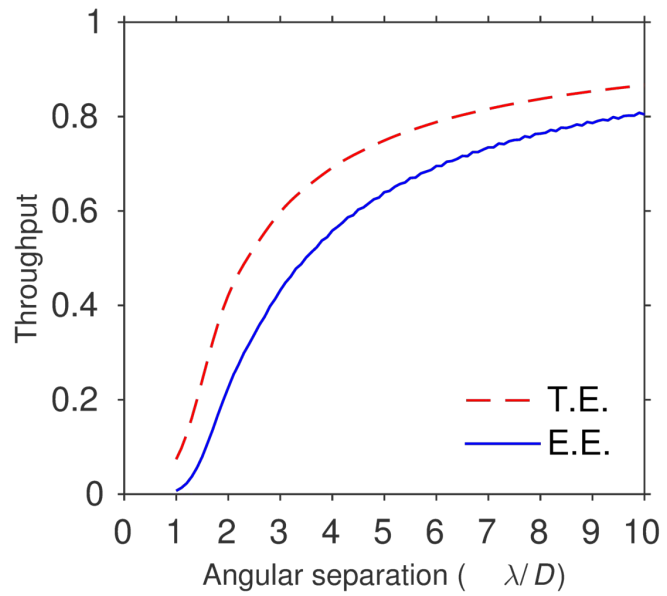

Figure 6. Relative throughput as a function of angular separation for an off-axis source for the Hex unobstructed aperture with DM solutions in Figure 4. 


\subsection{AFO Algorithm Solution for the LUVOIR'A" Aperture}

In Figures 7 and 8, we show the resulting beam-shaping solutions for an "ideal DM" for the LUVOIR architecture 'A' aperture. The "ideal DM" solutions are modeled as Optical Path Difference (OPD) phase masks in both the entrance pupil and Fresnel Plane. The entrance aperture was left unmodified (i.e. the outer perimeter of the aperture was not masked out with a smaller circular mask), and the Lyot stop (shown in the lower left of Figure 8) was a copy of the entrance pupil aperture with a circular mask covering the secondary mirror. No apodization was used in the optimization, with the $10^{-10}$ (normalized) contrast achieved purely with the phase solutions in the entrance and Fresnel planes. We emphasize that these coronagraph design solutions are for an on-axis point source. These solutions are a preliminary study of the feasibility of a vortex focal plane mask with a central obscured aperture such as that considered for the LUVOIR 'A" architecture. Work in progress is including robustness to the finite size of the star in the optimization, which must be taken into account for a primary mirror of 15 meters used for the LUVOIR mission concept.

We note that the use of "ideal DM's" here for LUVOIR is significant in demonstrating the potential throughput gains if the on-axis diffracted light is suppressed with pure phase beam shaping. One of the unique capabilities of the AFO algorithm is the ability to solve for phase variations at each "pixel", leading to a solutions of prohibitive dimension for algorithms such as stroke minimization EFC (relying on computation of the Jacobian for each DM degree of freedom as outlined $i^{7}$ ).

\section{SUMMARY AND CONCLUSIONS}

In summary, the goal of direct imaging of exoplanets in the habitable zone requires large (and therefore segmented) aperture telescopes together with some means of high-contrast imaging. We have developed a new "Auxiliary Field Optimization" (AFO) algorithm for coronagraph design for use with segmented apertures. Our initial focus in the development of the AFO algorithm has been to solve for either beam-shaping phase solutions (this paper), or apodization solutions ${ }^{3}$ (see also G. Ruane et. al., these proceedings) for a fixed focal plane mask and Lyot stop. Motivated by the excellent performance of the vortex focal plane mask for unobscured uniform circular aperture telescopes, we have applied the AFO algorithm to find solutions enabling the vortex coronagraph to be used with segmented aperture telescopes. We find viable solutions for the catalog of unobscured SCDA apertures (either beam shaping or apodization) which are insensitive to diffraction from a resolved stellar disc of 0.1 mas (we refer again to G. Ruane et. al. for a detailed sensitivity analysis). For the centrally obscured segmented apertures, we find sensitivity to the resolved stellar disc when solutions are optimized for suppression of a point on-axis source alone. We are currently working to build into the optimization the ability to suppress a resolved stellar disc with a generalization of the AFO algorithm to include optimization over the focal plane mask, conjugate pupil complex (or gray-scale amplitude only) mask, along with phase beam shaping with two DM's.

The AFO algorithm used for solving for the "ideal DM" solutions is also of interest in considering potential technology development in order to boost coronagraph performance. Novel devices such as Liquid Crystal Polymers are now being developed that show the "ideal DM" solutions might be realizable in hardware. These solutions are also valuable in providing insight into the spatial frequencies needed for pure phase beam-shaping solutions, and shed light into the potential trade-offs of conjugate plane apodization and beam-shaping. The former reduces throughput but is achromatic, while the latter results in higher throughput albeit over a restricted wavelength bandpass. Which design is best suited to a given mission concept requires detailed sensitivity analysis and end-to-end simulation including wavefront estimation and control, as closed-loop control (implemted with DM's) will introduce a wavelength dependence and limit the bandpass over which we can achieve reasonable signal to noise (balancing both contrast and planet throughput). 

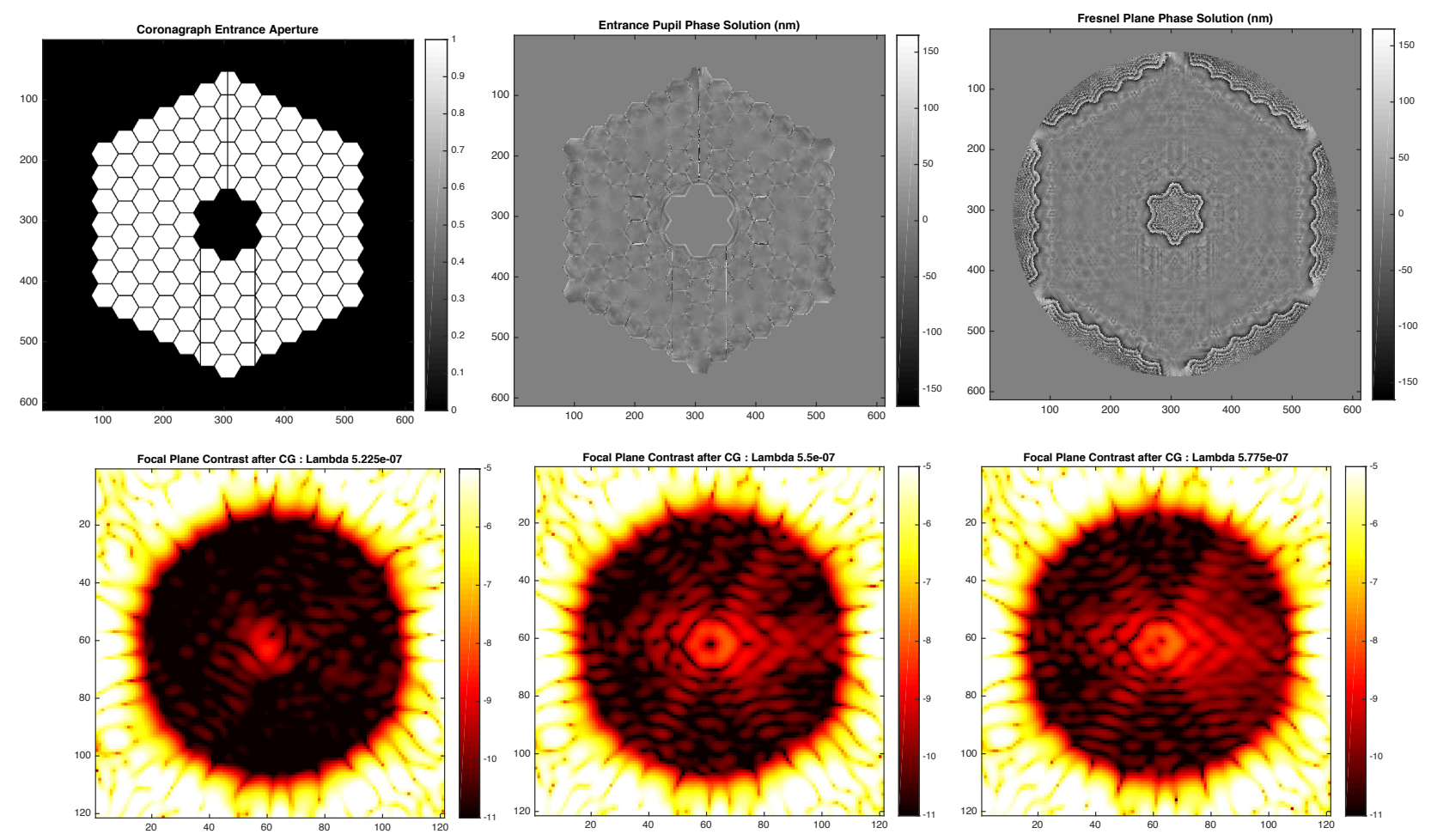

Figure 7. "Ideal DM" solutions for the LUVOIR "A" architecture, achieving $10^{-10}$ suppression in a $10 \%$ band (550 nm LUVOIR Aperture - Vortex Charge 4 and 6 Throughput Comparisons
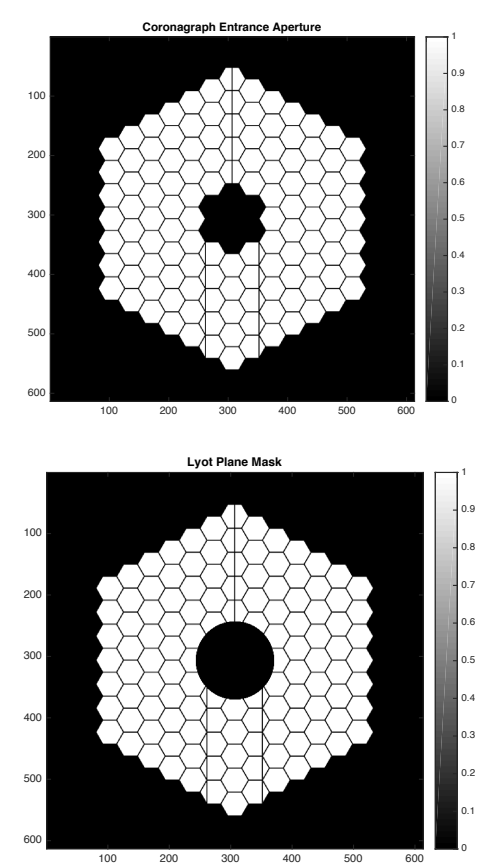
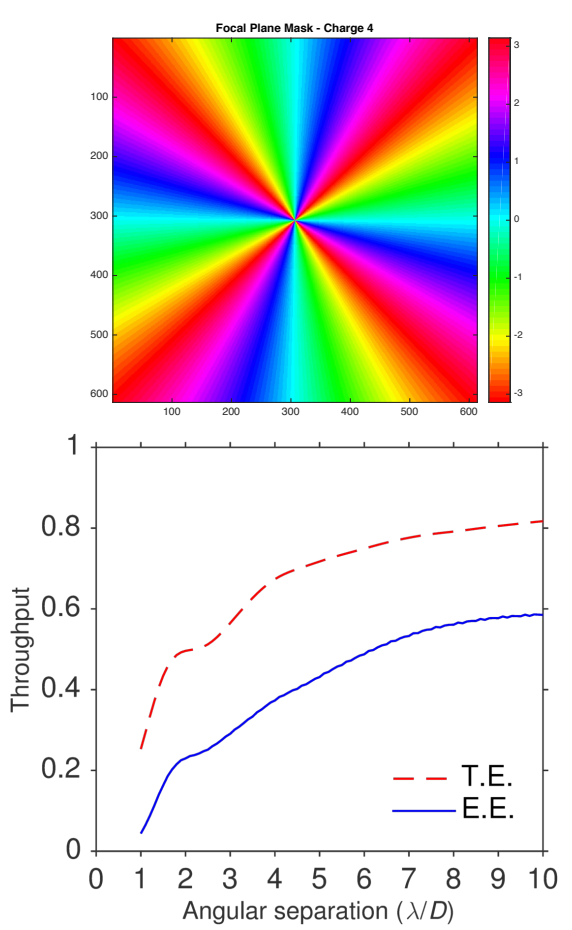

Beam-shaping, Charge 4
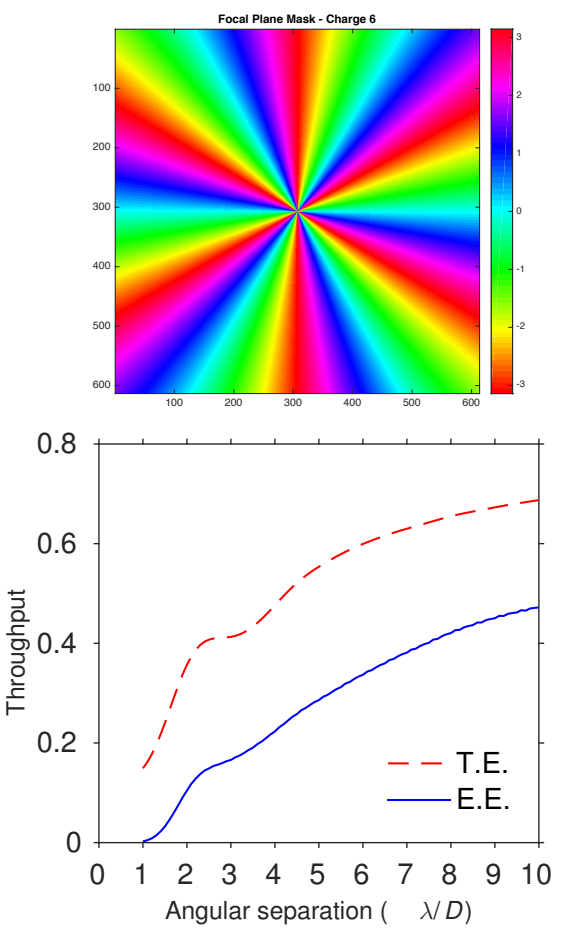

Beam-shaping, Charge 6

Figure 8. Comparison of relative throughput for "ideal DM" solutions for the LUVOIR "A" architecture, for a charge 4 and 6 vortex focal plane mask. Left top shows the entrance pupil aperture, and left bottom shows the Lyot stop. Both solutions are designed to achieve $10^{-10}$ suppression in a $10 \%$ band (550 $\mathrm{nm}$ central wavelength) of diffracted light for an on-axis point source. 


\section{APPENDIX A. CONVERGENCE OF THE AUXILIARY FIELD OPTIMIZATION ALGORITHM}

\section{A.1 Marginalized Objective for Coronagraph Design Variables}

The auxiliary field optimization algorithm is really a form of "expectation-maximization", in which we steadily improve our objective function which is associated with $-\log P[\Gamma, \Phi]$, the negative of the $\log$ of a probability density on the phase control degrees of freedom (or amplitude degrees of freedom - here we restrict the discussion to optimization of the deformable mirror phase degrees of freedom). As noted in the main discussion, the central idea is to embed our optimization problem into one containing additional auxiliary degrees of freedom to provide linear, convex subproblems which inform the variation of our coronagraph designs leading to good feasible solutions. In this appendix we want to explicitly show that our $-\log P$ converges in a limit to the original objective $\|Q C U(\Gamma, \Phi) X(0)\|^{2}$ (the dark hole energy of the on-axis source $X(0)$ ), so that our solutions in this sense gradually relax to local minima of the original problem. In other words, we show here that the auxiliary variables "integrate out" in the correct manner.

Our on-axis dark hole energy objective function is "promoted to a probability on DM solutions along with auxiliary variables"

$$
P[\Gamma, \Phi, W] \propto e^{-\beta\|Q C W\|^{2}-b\|W-U(\Gamma, \Phi) X(0)\|^{2}}
$$

where to review, $W$ is the fictitious auxiliary field in a conjugate pupil plane (downstream of the deformable mirrors but before the focal plane mask), $\Phi$ is the phase on an entrance pupil DM, and $\Gamma$ is the phase of a DM in the Fresnel plane.

We would like to check that the introduction of the auxiliary variables leads to a sensible objective function for the actual control degrees of freedom to be optimized, i.e. $(\Gamma, \Phi)$. For any value of the "inverse temperature" $\beta$ and regularization parameter $b$, we have an objective function for the design parameters given by $-\log P[\Gamma, \Phi]$, where $P[\Gamma, \Phi]$ is the measure integrated over the auxiliary variables $W$. We now analytically integrate over the auxiliary variables and show that in the limit $b \gg 1$ our auxiliary field marginalized objective $-\log P[\Gamma, \Phi]$ converges to the dark hole energy for the on-axis source.

To quickly integrate out the auxiliary variables we note that

$$
\begin{aligned}
P[\Gamma, \Phi] & \propto \int d W e^{-\beta\|Q C W\|^{2}-b\|W-U(\Gamma, \Phi) X(0)\|^{2}} \\
& =e^{-\beta\|Q C \hat{W}\|^{2}-b\|\hat{W}-U(\Gamma, \Phi) X(0)\|^{2}} \int d W e^{-(W-\hat{W})^{\dagger}\left(\beta C^{\dagger} Q C+b I\right)(W-\hat{W})}
\end{aligned}
$$

where in the second line we evaluate the joint measure at the field

$$
\hat{W}=\left(b I+\beta C^{\dagger} Q C\right)^{-1} b U(\Gamma, \Phi) X(0)
$$

(note $-\hat{W}$ is a function of the DM control degrees of freedom). The integral in Equation 38 is now readily seen to be a well-known Gaussian integral, and shifting the integration $d W \rightarrow d(W-\hat{W})$ leads to a $(\Gamma, \Phi)$ independent factor,

$$
\begin{aligned}
P[\Gamma, \Phi] & \propto \int d W e^{-\beta\|Q C W\|^{2}-b\|W-U(\Gamma, \Phi) X(0)\|^{2}} \\
& \propto e^{-\beta\|Q C \hat{W}\|^{2}-b\|\hat{W}-U(\Gamma, \Phi) X(0)\|^{2}}\left|b I+\beta C^{\dagger} Q C\right|^{-1 / 2}
\end{aligned}
$$

Our auxiliary field marginalized objective function is therefore

$$
-\log P[\Gamma, \Phi] \sim \beta\|Q C \hat{W}(\Gamma, \Phi)\|^{2}+b\|\hat{W}(\Gamma, \Phi)-U(\Gamma, \Phi) X(0)\|^{2}
$$

where we emphasize that the terms on the right are entirely determined by $(\Gamma, \Phi)$. Using the functional relation of $\hat{W}(\Gamma, \Phi)$ in Equation 39, we can simplify this expression

$$
-\log P[\Gamma, \Phi] \sim \beta X^{\dagger}(0) U^{\dagger}(\Gamma, \Phi) C^{\dagger} Q\left[I+\left(b^{-1} \beta\right) Q C C^{\dagger} Q\right]^{-1} Q C U(\Gamma, \Phi) X(0)
$$


This is the implicit objective function for our coronagraph design variables for any values of $(\beta, b)$, and the form which is non-decreasing during AFO iterations. We now take the $b \gg 1$ limit, equivalent to a very strong penalty for the auxiliary field to deviate from the true physical conjugate pupil field. In the $b \gg 1$ limit we have

$$
\begin{array}{cl}
-\log P[\Gamma, \Phi] & \rightarrow_{b \gg 1} \quad \beta X^{\dagger}(0) U^{\dagger}(\Gamma, \Phi) C^{\dagger} Q C U(\Gamma, \Phi) X(0) \\
& =\quad \beta\|Q C U(\Gamma, \Phi) X(0)\|^{2}
\end{array}
$$

showing that our auxiliary field marginalized objective converges to the on-axis source dark hole energy as intended.

We note that as we proceed with AFO iterations, we should gradually be increasing the regularization parameter $b$. In practice, we iterate for several steps, and then check if increasing $b$ gives a greater improvement in contrast. If so, we switch to a larger value and continue.

\section{A.2 Convergence of AFO Iterations to Local Minima}

We now show that AFO iterations always result in a steady non-decreasing sequence of our marginalized objective $-\log P[\Gamma, \Phi]$. We show this by deriving an Expectation-Maximization algorithm for our design variables $(\Gamma, \Phi)$ using the joint measure $P[\Gamma, \Phi, W]$.

We note that the conditional probability measure $P[W \mid \Gamma, \Phi]$ is Gaussian with a "peak" at the field

$$
\max _{W}(-\log P[W \mid \Gamma, \Phi])=\left(b I+C^{\dagger} Q C\right)^{-1} b U(\Gamma, \Phi) X(0)
$$

Jensen's inequality leads to the well known Expectation-Maximization iterative algorithm ${ }^{9}$

$$
\begin{aligned}
\log \left(\frac{P[\Gamma, \Phi]}{P\left[\Gamma_{n}, \Phi_{n}\right]}\right) & \geq \int d W P\left[W \mid \Gamma_{n}, \Phi_{n}\right] \log \left(\frac{P[\Gamma, \Phi \mid W]}{P\left[\Gamma_{n}, \Phi_{n} \mid W\right]}\right) \\
& =-\int d W P\left[W \mid \Gamma_{n}, \Phi_{n}\right]\left(\|W-U(\Gamma, \Phi) X(0)\|^{2}-\left\|W-U\left(\Gamma_{n}, \Phi_{n}\right) X(0)\right\|^{2}\right)
\end{aligned}
$$

The above shows that maximizing the right-hand side never results in a worse objective $-\log P$. For the Gaussian (conditioned on $\left.\left(\Gamma_{n}, \Phi_{n}\right)\right)$ maximizing the right-hand side is equivalent to

$$
\left\{\Gamma_{n+1}, \Phi_{n+1}\right\}=\min _{\Gamma, \Phi}\left(\left\|\hat{W}\left(\Gamma_{n}, \Phi_{n}\right)-\left(P_{\lambda}^{\dagger} e^{i\left(\lambda_{0} / \lambda\right) \Gamma} P_{\lambda} e^{i\left(\lambda_{0} / \lambda\right) \Phi}\right) X(0)\right\|^{2}\right)
$$

with $\hat{W}\left(\Gamma_{n}, \Phi_{n}\right)$ the mean field given the past DM solutions.

\section{APPENDIX B. OPTIMIZATION OF DEFORMABLE MIRROR INFLUENCE FUNCTION ACTUATOR HEIGHTS}

We now generalize the phase variations in Equation 29 for the case of optimizing DM actuator heights instead of the phase at every 'pixel'. The phase generated by a DM is model as a linear combination of influence functions

$$
\begin{aligned}
\Gamma & =4 \pi \lambda_{0}^{-1} \sum_{k} h_{k}(p) d_{k} \\
\Phi & =4 \pi \lambda_{0}^{-1} \sum_{k} h_{k}(p) c_{k}
\end{aligned}
$$

where $\left\{c_{k}, d_{k}\right\}$ are the actuator heights (in physical units) and $h_{k}(p)$ is the $k$-th influence function (at pixel $p$ ). In matrix form then

$$
\begin{aligned}
& \Gamma=H d \\
& \Phi=H c
\end{aligned}
$$


where we absorb the $4 \pi \lambda_{0}^{-1}$ in the columns of $H$.

As before we want to solve

$$
\begin{aligned}
\delta c & =\min _{\delta c}\left(\int d \lambda\left\|W_{\lambda}-P_{\lambda}^{\dagger} e^{i\left(\lambda_{0} / \lambda\right) \Gamma} P_{\lambda} e^{i\left(\lambda_{0} / \lambda\right) \Phi} e^{i\left(\lambda_{0} / \lambda\right) H \delta c} X(0)\right\|^{2}\right) \\
& =\min _{\delta \Phi}\left(\int d \lambda\left\|\left(e^{-i \Phi\left(\lambda_{0} / \lambda\right)} P_{\lambda}^{\dagger} e^{-i \Gamma\left(\lambda_{0} / \lambda\right)} P_{\lambda} W_{\lambda}\right)-e^{i\left(\lambda_{0} / \lambda\right) H \delta c} X(0)\right\|^{2}\right)
\end{aligned}
$$

(where for notational convenience we only explicitly referred to the DM actuator variations being considered, and $\Phi$ and $\Gamma$ are determined by the current DM actuator settings as above).

As before we again define

$$
Z_{\lambda}(\Gamma)=e^{-i \Phi\left(\lambda_{0} / \lambda\right)} P_{\lambda}^{\dagger} e^{-i \Gamma\left(\lambda_{0} / \lambda\right)} P_{\lambda} W
$$

so that our minimization problem reduces to

$$
\delta \Phi=\min _{\delta \Phi}\left[\int d \lambda\left(Z_{\lambda}^{*}(\Gamma)-e^{-i\left(\lambda_{0} / \lambda\right) H \delta c} X(0)^{*}\right)\left(Z_{\lambda}(\Gamma)-e^{i\left(\lambda_{0} / \lambda\right) H \delta c} X(0)\right)\right]
$$

Setting the variation of the quadratic form above with respect to the actuator heights $\delta c$ to zero (i.e. taking the gradient of the above with respect to $\delta c$ and setting to 0 ) leads to the condition

$$
0=H^{T} \cdot \int d \lambda\left(\frac{\lambda_{0}}{\lambda}\right)\left(Z_{\lambda}(\Gamma) e^{-i\left(\lambda_{0} / \lambda\right) H \delta c} X(0)^{*}-Z_{\lambda}^{*}(\Gamma) e^{i\left(\lambda_{0} / \lambda\right) H \delta c} X(0)\right)
$$

(where $H^{T}$ is the transpose of the DM basis function response matrix). As before, we define the modulus and phase at each wavelength

$$
Z_{\lambda}(\Gamma) X(0)^{*} \equiv\left|Z_{\lambda}(\Gamma) X(0)^{*}\right| e^{i \Psi_{\lambda}}
$$

Therefore, the optimal DM variation satisfies

$$
\begin{aligned}
0 & =H^{T} \cdot \int d \lambda\left(\frac{\lambda_{0}}{\lambda}\right)\left|Z_{\lambda}(\Gamma) X(0)^{*}\right|\left(e^{i \Psi_{\lambda}} e^{-i\left(\lambda_{0} / \lambda\right) H \delta c}-e^{-i \Psi_{\lambda}} e^{i\left(\lambda_{0} / \lambda\right) H \delta c}\right) \\
& =(2 i) H^{T} \cdot \int d \lambda\left(\frac{\lambda_{0}}{\lambda}\right)\left|Z_{\lambda}(\Gamma) X(0)^{*}\right| \sin \left[\Psi_{\lambda}-\left(\lambda_{0} / \lambda\right) H \delta c\right] \\
& \approx(2 i) H^{T} \cdot \int d \lambda\left(\frac{\lambda_{0}}{\lambda}\right)\left|Z_{\lambda}(\Gamma) X(0)^{*}\right|\left(\Psi_{\lambda}-\left(\lambda_{0} / \lambda\right) H \delta c\right)
\end{aligned}
$$

(where in the last line we again approximate $\sin (x) \approx x$ ). The above gives a linear relation between the DM variations and a amplitude modulated source term from the $\Psi_{\lambda}$,

$$
\delta c=\left[\int d \lambda\left(\frac{\lambda_{0}}{\lambda}\right)^{2} H^{T}\left|Z_{\lambda} X(0)^{*}\right| H\right]^{-1}\left(\int d \lambda\left(\frac{\lambda_{0}}{\lambda}\right) H^{T}\left|Z_{\lambda} X(0)^{*}\right| \Psi_{\lambda}\right)
$$

\section{ACKNOWLEDGMENTS}

We thank Matthew Bolcar (NASA Goddard Space Flight Center) for providing the aperture for the LUVOIR architecture 'A' design used in this paper. This work was partially performed at the Jet Propulsion Laboratory, California Institute of Technology, under contract with the National Aeronautics and Space Adminstration. Government sponsorship acknowledged. Copyright, 2017, all rights reserved. 


\section{REFERENCES}

[1] Stark, C., Roberge, A., Mandell, A., and Robinson, T., "Maximizing the ExoEarth Candidate Yield From A Future Direct Imaging Mission," Astrophys. J. 795, 122-141 (2014).

[2] Mawet, D., Riaud, P., Absil, O., and Surdej, J., "Annular Groove Phase Mask Coronagraph," Astrophys. J. 633, 1191-1200 (2005).

[3] Ruane, G., Jewell, J., Mawet, D., Pueyo, L., and Shaklan, S., "Apodized vortex coronagraph designs for segmented aperture telescopes," Proc. SPIE 9912, 9912L (2016).

[4] Siegler, N. and Shaklan, S., "Segmented Coronagraph Design and Analysis Task Description." Available at http://exep.jpl.nasa.gov/files/exep/SCDA_Summary.pdf (2016).

[5] Feinberg, L., Hull, T., and Knight, J., "Apertures for Segmented Coronagraph Design and Analysis." Available at http://exep.jpl.nasa.gov/files/exep/SCDAApertureDocument0504161.pdf (2016).

[6] Boyd, S. and Vandenberghe, L., [Convex Optimization], Cambridge University Press, Cambridge, U.K. (2009).

[7] Groff, T., Riggs, A. E., Kern, B., and Kasdin, N. J. ., "Methods and limitations of focal plane sensing, estimation, and control in high-contrast imaging," J. Astron. Telesc. Instrum. Syst. 2(1), 011009 (2016).

[8] Mazoyer, J., Pueyo, L., N'Diaye, M., and et. al., "Correcting for the effects of pupil discontinuities with the ACAD method," Proc. SPIE 9904, 9904T-1 (2016).

[9] Dempster, A., Laird, N. M., and Rubin, D., "Maximum Likelihood from Incomplete Data via the EM Algorithm," J. R. Stat. Soc. B 39(1), 1-38 (1977). 